\title{
Video Motion Analysis Techniques: A Comparative Study
}

\author{
Samidha Vengurlekar ${ }^{1}$, Dipti Jadhav ${ }^{2}$ \\ M.E. Student, Information Technology Department, RAIT, Nerul Navi Mumbai, India ${ }^{1}$ \\ Associate Professor, Information Technology Department, RAIT, Nerul Navi Mumbai, India ${ }^{2}$
}

\begin{abstract}
Video analysis gives the detailed information about moving objects from the video. It is of great importance in the fields of sports, collision detection on road and rail routes, speed and acceleration calculation and many other fields. In this paper, we have compared four systems based on different algorithms and applied for different domains. First technique is for basketball video analysis, where semantic event extraction, slow motion replay detection methods have been introduced. Second, fire and smoke detection algorithm has been introduced. Third, the system uses surveillance cameras to detect the traffic state. Fourth, obstacle detection based railroad collision warning system. All these systems are diverse in nature with different application areas. The comparison showed that the system for sports have gained highest accuracy of all the other systems.
\end{abstract}

Keywords: Sports video analysis, fire and smoke detection, railroad obstacle detection, traffic state detection.

\section{INTRODUCTION}

Network technology has led a quick development in acquiring videos on demand on a greater scale. The mobile devices are mostly very useful for viewing videos on a greater extent anytime anywhere. Many videos from various fields are generated and getting viral on everyday basis. These videos are heavy in sizes and hence it becomes impossible to see all of them. So, all the important events or highlights are mostly seen so as to reduce time. Only partial videos or certain segments are of preferable importance. Especially in sports, the viewers are interested only in watching important sports segments or highlights rather than entire match. Hence, analysis of sports video, such as semantic event extraction and slow motion replay detection, has become a valuable research topic [1].

Economy growth led to increase in high and large number of buildings.If fire breaks out in any of the building with complex structure and huge number of staff may cause extensive damage of property and staff. It may cause bad social impact. For fire extinguishing and evacuation of staff many systems have been developed but the fire alarm gives alert when the fire has caused much damage. So technically there was a need of a fire and smoke detection technique to bring the situation under control. In large constructions, many systems fail to rightly detect fire and smoke and cannot meet the demand of early fire detection in these places. In comparison toconventional fire detectors, detection of fire through video has advantages such as, accurate and fast detection, large protection area particularly applicable to large rooms and high buildings [2].

Video analysis has also gained importance in traffic state detection. The traffic data has been collected and it is classified into categories such as congestion, slow, smooth and accident. To detect such information, image processing plays an important role. With the use of Image processing, stills from video can be extracted to check the traffic state. Firstly, special cameras where assigned for such detection but now cameras used for surveillance can also be applied for this purpose. Such a system is very useful in route decision making, traffic flow planning, etc. [3].

With increase of railroads, there also have increased risks of accidents. Many railway crossings are not well-equipped which can notify the arrival of train from any of the end. It is managed manually for many small places. Hence the use of video made it easy for train drivers to get warning signals if any object is detected in the rail route. The system need to be very alert to detect objects and notify drivers accordingly [4].

\section{APPLICATION OF VIDEO ANANLYSIS}

Video analysis is applicable in many fields; the list is as generated below.

- Video surveillance: The video surveillance holds a great impact in security. We mostly see its applications on areas where much security is needed. Example, airports, passport offices, visa offices, parliament and other important places.

- Social media and the Internet: Most of the people in today's worlds use social media to share videos. Video analysis becomes very important to check the video before it gets uploaded and viral. 
- Virtual reality: If information is collected from videos of natural scenery and combined into the translation process then virtual environments can be very realistic.

- Vision-based robotics: In a complex zone such as disaster prone areas, robots well-equipped with cameras play very important roles. Robots may be used here for tracking and recognition of objects. Video processing of the data required for cameras of robots help in extracting some useful information.

- Biomedical applications: Video processing and analysis helps medical practitioners in diagnosing various diseases. The data obtained can be used for deciding the complexity level of diseases.

\section{OVERVIEW OF VIDEO ANALYSIS TECHNIQUES}

Video Analysis gains lot of importance in several fields where it becomes very critical to take decisions spontaneously. Some techniques have been discussed below:

\section{A. Semantic Event and Slow Motion Replay Extraction}

Semantic event extraction and slow motion extraction is an essential research work to analyze sports related videos where accurate decisions have to be made in very small time. Chun-Min et al. [1] proposed a framework to analyze basketball videos. The work is very concrete where scoreboard information is also extracted from the videos. Sports videos consist of two types of videos, one regular video which contains scoreboard and another replay video without a scoreboard. Hence the extraction process starts from categorizing the frames in two parts, scoreboard frame and nonscoreboard frame. It means that the sematic events appear during scoreboard frames and slow motion appears during replay videos or non-scoreboard frames. Slow motion replays take place only during temporal pausing of a basketball game, which consists of non-scoreboard frames only. This extraction is useful for further processing of videos.

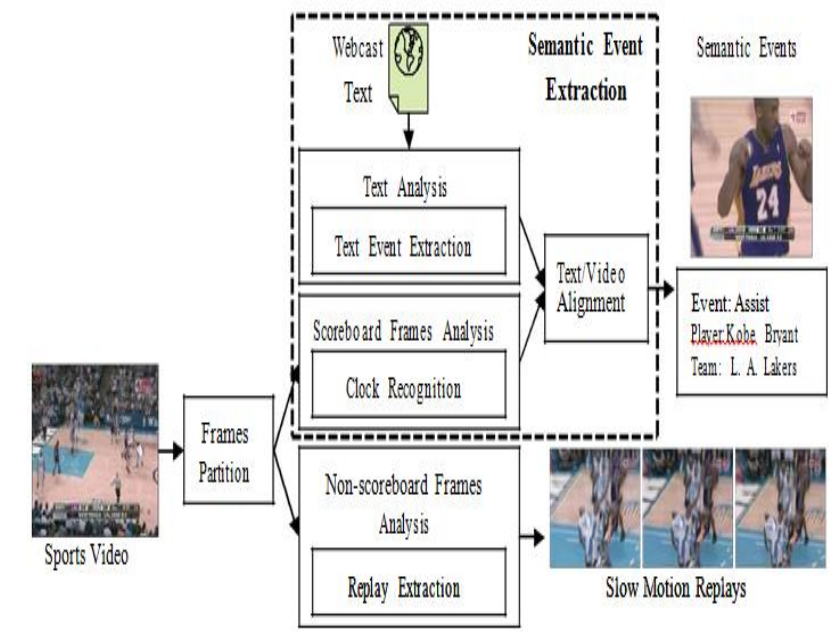

Fig.1. Semantic events and Slow motion replay extraction [1]

The framework shown in Fig. 1 finds both the types of frames in very effective manner. It takes a basketball video as an input. The frames are first partitioned into two categories: scoreboard frame and non-scoreboard frames. Then for each of the extracted frames, the simultaneous process is carried out. For scoreboard frame, semantic event extraction algorithm is applied where text in scoreboard is analyzed. Also the clock of the video is analyzed. Then on the basis of text and video alignment, semantic events are extracted. Similarly from non-scoreboard frames, replays and slow motion events are extracted. Other similar works have been done from past so many years [5]-[7], but the proposed system by ChunMin has given 100\% accuracy.

\section{B. Fire and Smoke Detection}

Yu et al. [2] designed a system for fire and smoke detection. They presented the framework which works in four stages: First, Fame differential method presented by Collins et al. [8] is used to detect and extract moving pixels and regions from the video. Second, color models are used to distinguish fire and smoke region. Two models for this detection are used. For fire, a color model consisting of red to orange and yellow colors is used. On the contrary, a model with shades of gray color is used to detect a smoke in the video. Third, foreground accumulation images are built of both flame and smoke. In the last stage, motion features of fire flame and smoke are each calculated based on block image processing and optical flow technique. Fig.2 shows the flow of entire system for fire and smoke detection. Video sequences are the input to the system. It alarms only fire and only smoke or both fire and smoke is detected in the video. 


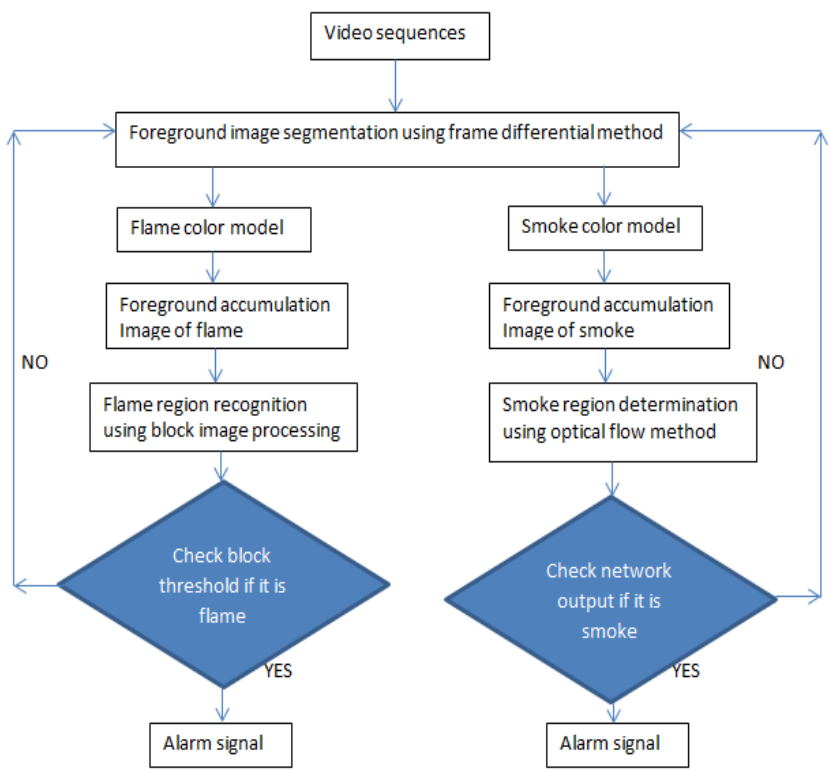

Fig. 2. Fire and Smoke Detection System Flowchart[2]

\section{Traffic State Detection}

Road Areas Extraction and Motion Detection: Extraction of road areas is directly related to road occupancy calculation. It helps in calculating important parameters like road width, moving objects on road, etc. Using traffic surveillance cameras, the road areas can be extracted by detecting regions without any change and vehicles can be detected as moving objects i.e. regions with several changes [9].In this, way region extraction is useful to find road areas in a video. For a moving vehicle, frame region is focused to check its difference from previous and next and then consecutive frames.

Road Space Occupancy Estimation: Edge Density of Road Areas: Road occupancy includes time occupancy and space occupancy. Time occupancy can be calculated as number of vehicles passed a section of a frame in a particular period of time. This can be calculated using a virtual loop in the video. Road space occupancy refers to the percentage of road area used. It is calculated as the ratio of vehicles projective area divided by the road area at a moment and can be also be called as vehicle density on road at thatpoint of time. Road time occupancy is generally directly proportional to road space occupancy. Vehicle region is a set of features described in an image. These features include edge, intensity, color, illumination, etc. If the vehicle density on road is high, then density of vehicle's feature on road also reaches a high level. It is quite difficult to observe and detect an individual vehicle in the video. For this purpose a density of image features is extracted to find the density of vehicle on the road. Illumination is one of the key features that helps in vehicle detection but it may keep on changing. While, edge is a robust image feature with few change. Hence road space occupancy can be obtained by edge pixels' density on road areas. For the extraction of vehicle edge map in road areas, Sobel algorithm is used[3].

Traffic Flow Speed Estimation: On detection of vehicle on the road in a video, the traffic flow speed has to be determined. The traffic flow vector is used to get the traffic flow speed. If many vehicles are detected in the video then the flow can be concluded to be congested. Similarly if all the vehicles are moving with particular speed then the flow can be referred to as smooth[3].

\section{Railroad Collision Warning}

Train route has to be secured from danger of accident. For this purpose, a train has to be ensemble with a camera for taking videos in order to detect obstacles posing danger to the train traveling. There are two strategies to deal with this. The first strategy detects the obstacles that are on the region near the rails. Fig. 3 shows a general diagram of this part of the system. Video captured from the view of a train driver is given as an input to the system. Then the video is processed in a frame to frame basis. At the very first stage, a filter is applied to improve the contours of the frames. Then a gray scale conversion and dimensioning reduction phase take place[4].

To represent the rails lines in the frames, Hough transform mechanism was used. It was observed that use of smaller image improves the process speed without affecting precision. Hence this system first resizes the image and eliminates the background objects. After this process and applying Hough transform algorithm the rail route lines were clearly found.

For the obstacle detection stage, canny algorithm is used first. Then the images are fit to a rectangular mask so as to pass on for further processing. The background objects are filtered to concentrate on rail lines. Small and disconnected objects are then eliminated. A sovereign tracing in each rail is led by using a small dynamic area that moves from the 
bottom of the train to the upper part. In each processing step, the area and centroid are calculated. When the metrics are far from the threshold values, the selected area grows and modified its centroid with the intention of enclosing the strange object.

Another strategy to detect rail obstacle is based on tracking objects that may cause danger to the rail but are far away from the route yet, a strategy based on the optical flow is used. Fig. 4 shows a scheme of the steps followed for detecting and tracking these obstacles[4].

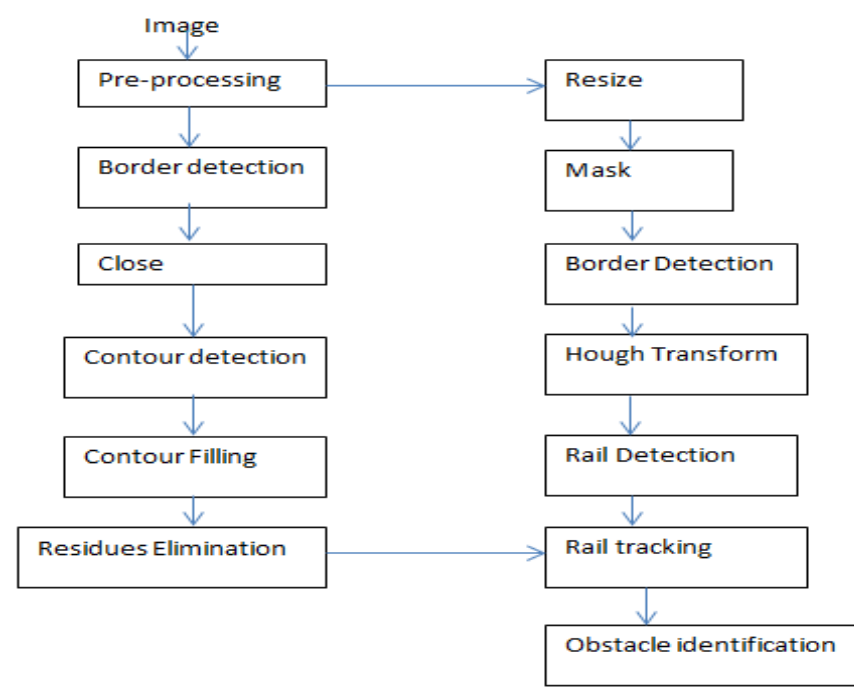

Fig. 3. Tracking Nearer Obstacles [4]

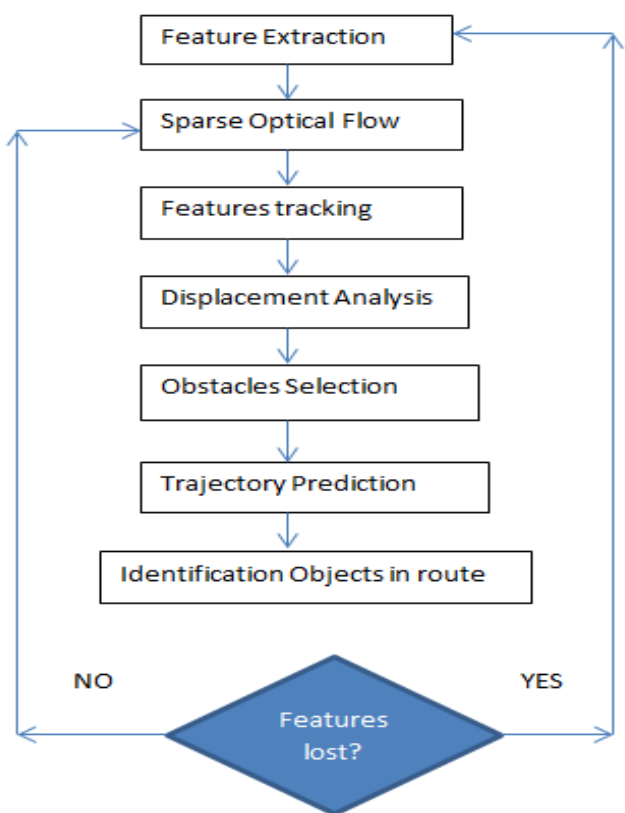

Fig. 4. Tracking Far away Obstacles [4]

\section{COMPARISON OF VIDEO ANALYSIS TECHNIQUE}

In the sports video the methods used are Video frame partition, semantic event extractor and replay detection which gave very high accuracy and have advantages like increased performance and detection rate. Rail video recording used methods like Hough Transform, Optical Flow which gave moderate accuracy and Detection of fixed objects is the advantage. The video of Traffic surveillance camera have methods like Traffic state detection and Traffic flow speed estimation which gave high accuracy only in day time. The Real time video with fire and smoke used methods like Video fire detection, Optical flow Smoke determination and the advantage is Realize real time fire detection for three cases: Fire with flame and none smoke. Fire with smoke and none flame. Fire with both flame and smoke. 
Vol. 6, Issue 4, April 2017

\begin{tabular}{|c|c|c|c|c|}
\hline $\begin{array}{c}\text { NAME OF } \\
\text { THE } \\
\text { AUTHOR } \\
\end{array}$ & $\begin{array}{l}\text { Chun-Min Chen and } \\
\text { Ling-Hweichen[1] }\end{array}$ & $\begin{array}{l}\text { Jonny A. Uribe, Luis } \\
\text { Fonseca, J.F.Vargas[4] }\end{array}$ & $\begin{array}{c}\text { Xiying Li, Yongye } \\
\text { she, DonghuaLuo, Zhi } \\
\text { Yu[3] }\end{array}$ & $\begin{array}{c}\text { Chunyu Yu, Zhibin Mei, } \\
\text { Xi Zhang[2] }\end{array}$ \\
\hline $\begin{array}{c}\text { METHOD } \\
\text { PROPOSED }\end{array}$ & $\begin{array}{l}\text { Video frame partition. } \\
\text { Semantic event } \\
\text { extractor, } \\
\text { Replay detection. }\end{array}$ & $\begin{array}{l}\text { Hough Transform. } \\
\text { Optical Flow. }\end{array}$ & $\begin{array}{c}\text { Traffic state detection. } \\
\text { Traffic flow speed } \\
\text { estimation. }\end{array}$ & $\begin{array}{l}\text { Video fire detection. } \\
\text { Optical flow Smoke } \\
\text { determination. }\end{array}$ \\
\hline $\begin{array}{l}\text { TYPE OF } \\
\text { VIDEO }\end{array}$ & Sports video. & $\begin{array}{l}\text { Railroad video } \\
\text { recording. }\end{array}$ & $\begin{array}{c}\text { Traffic surveillance } \\
\text { camera. }\end{array}$ & $\begin{array}{l}\text { Real time video with fire } \\
\text { and smoke. }\end{array}$ \\
\hline ACCURACY & Very High accuracy & Moderate accuracy & $\begin{array}{l}\text { High accuracy in day } \\
\text { time. }\end{array}$ & High accuracy \\
\hline $\begin{array}{l}\text { ADVANTAG } \\
\text { ES }\end{array}$ & $\begin{array}{c}\text { Performance and } \\
\text { detection rate } \\
\text { increased. } \\
\text { Detection rate reaches } \\
100 \% .\end{array}$ & $\begin{array}{l}\text { Detection of fixed } \\
\text { objects. }\end{array}$ & $\begin{array}{l}\text { High accuracy in day } \\
\text { time. }\end{array}$ & $\begin{array}{c}\text { Realize real time fire } \\
\text { detection for three cases: } \\
\text { Fire with flame and } \\
\text { none smoke. Fire with } \\
\text { smoke and none flame. } \\
\text { Fire with both flame and } \\
\text { smoke. }\end{array}$ \\
\hline $\begin{array}{c}\text { FUTURE } \\
\text { SUGGESTIO } \\
\mathbf{N}\end{array}$ & $\begin{array}{l}\text { No basketball specific } \\
\text { features are used so it } \\
\text { is expectable to push } \\
\text { forward the framework } \\
\text { to other sports. }\end{array}$ & $\begin{array}{l}\text { Robustness of the } \\
\text { algorithm to changes in } \\
\text { light and weather } \\
\text { conditions. } \\
\text { Correct identification } \\
\text { of rails. } \\
\text { To detect signs, } \\
\text { tunnels, bridges etc. }\end{array}$ & $\begin{array}{l}\text { Thresholds by self- } \\
\text { calibration. }\end{array}$ & $\begin{array}{l}\text { More improvement on } \\
\text { other kind of disturbance } \\
\text { like lights turning on } \\
\text { and off frequently. }\end{array}$ \\
\hline
\end{tabular}

\section{CONCLUSIONS}

Video analysis is an important factor when it comes on different application which highly belongs on it. Analyzing some things based on different factors is very critical. There are different techniques which helped the analyzing task very easily. In different applications like railway, traffic state detection, sports videos analysis, flames and smoke analysis there are different methods and techniques to analyses the video. The methods discussed in paper include use of Hough transform, Optical flow, semantic extractor, fire detection, smoke determination. In this paper we have discussed different techniques for video motion analysis based on various parameters. The various parameters discussed in this paper are method proposed, type of video, accuracy, advantages and future suggestions. These techniques helped to analyses the video motion to complete the task of the application.

\section{ACKNOWLEDGMENT}

I am thankful to the authors and my guide for inspiring me to research further in video analysis domain.

\section{REFERENCES}

[1] Chun-Min Chen and Ling-Hwei Chen, "Novel Framework For Sports Video Analysis: A Basketball Case Study," IEEE International Conference on Image Processing (ICIP), pp. 961-965, October 2014.

[2] Chunyu Yu, Zhibin Mei, Xi Zhang, "A real-time video fire flame and smoke detection algorithm," Asia-Oceania Symposium on Fire Science and Technology, vol. 9, pp.891-898, 2013.

[3] Xiying Li, Yongye She, Donghua Luob, Zhi Yu, “A Traffic State Detection Tool for Freeway Video Surveillance System,” COTA International Conference of Transportation Professionals, vol. 13, pp.2453-2461, 2013.

[4] Jonny A. Uribe, Luis Fonseca, J. F. Vargas, "Video Based System for Railroad Collision Warning,"2012 IEEE International Carnahan Conference on Security Technology (ICCST), pp.280-285, 2012.

[5] Y. H. Chen and L. Y. Deng, "Event mining and indexing in basketball video," in Int. Conf. on Genetic and EvolutionaryComputing (ICGEC), pp. 247-251, Aug. 29-Sep. 1, 2011.

[6] C. Xu, J. Wang, K. Wan, Y. Li, and L. Duan, "Live sports event detection based on broadcast video and web-casting text," in Proc. ACM Int. Conf. on Multimedia (MM '06), pp. 221-230, 2006.

[7] P. Lin, S. Li, T. Tsai, and Y. Tsai, "Tagging webcast text in baseball videos by video segmentation and text alignment," IEEETrans. Circuits Syst. Video Technol., vol. 22, no. 7, pp. 999-1013,2012.

[8] Collins, R., Lipton, A., Kanade, T., A System for Video Surveillance and Monitoring. Tech. Report CMU-R1-TR-00-12, Robotics Institute, Carnegie Mellon University, 2000.

[9] Xiying Li, Yongye She, Guigen Yang, Youting Zhao, Donghua Luo (2011). A Traffic Congestion Detection Method for Surveillance Videos Based on Macro Optical Flow Velocity. In The 11th International Conference of Chinese Transportation Professionals (ICCTP2011). CDROM. Nanjing, China, 2011, pp. 1569-1578. 